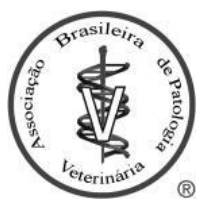

Case Report

\title{
Metastatic papillary mesothelioma in a dog: a case report
}

\author{
Érica A. Viscone ${ }^{1 *}$, Alessandra C. Rodrigues ${ }^{2}$, Fernanda G. Menegon ${ }^{3}$, Sofia Borin-Crivellenti ${ }^{3}$, \\ Márcio de B. Bandarra ${ }^{1}$
}

\begin{abstract}
'Animal Pathology Laboratory, Veterinary Hospital of Universidade Federal de Uberlândia, Uberlândia - MG, Brazil.
${ }^{2}$ Applied Immunology and Parasitology Laboratory, Universidade Federal de Uberlândia, Uberlândia - MG, Brazil.

${ }^{3}$ Small Animal Internal Medicine Service, Veterinary Hospital of Universidade Federal de Uberlândia - MG, Brazil. *Corresponding author: E-mail: ericaviscone@gmail.com
\end{abstract}

Submitted May, $26^{\text {th }}$ 2019, Accepted August, $20^{\text {th }} 2019$

\begin{abstract}
A six-year-old female Dachshund presented intense abdominal distension caused by hemorrhagic effusion. Cytological examination of the fluid was performed and suggested a carcinoma. The animal was submitted to exploratory laparotomy and histological examination of the substantially altered spleen revealed epithelioid mesothelioma. Owing to a poor prognosis, poor response to chemotherapy, and development of thoracic effusions that required daily drainages, the owners decided for euthanasia. At necropsy, besides a $600 \mathrm{~mL}$ of sera-hemorrhagic abdominal fluid, no abnormal gross finding was identified in the abdominal cavity. The thoracic cavity was also filled with approximately $200 \mathrm{~mL}$ of effusion with same features as those detected in the abdominal fluid. A few firm white-to-gray nodules, with sizes ranging from 0.1 to $0.3 \mathrm{~cm}$, were found in the lungs and diaphragm. Samples of the nodules were stained for routine histopathology and submitted to immunohistochemistry (IHC) assays using the antibodies vimentin, PAN Cytokeratin, calretinin, and TTF-1. Histopathology and IHC findings confirmed the diagnosis of metastatic papillary mesothelioma.
\end{abstract}

Key words: mesothelioma, immunohistochemistry, malignant neoplasia, cytology.

\section{Introduction}

Mesothelioma is an uncommon tumor that arises from serous linings cells of the pericardial, pleural, and peritoneal cavities, and that may affect all three locations simultaneously. The malignancy can be presented as villus projections on a thickened mesentery or serosal surface (9), and the neoplastic mesothelial cells can show either epithelial- or mesenchymal-like differentiation. Mesotheliomas are subclassified as epithelioid, sarcomatous (fibrous), or mixed (6). In humans, epithelioid mesotheliomas are further subdivided into one up to 17 different histologic subtypes, including papillary, tubular, solid, sclerosing, cystic, and deciduoid subtypes (6). Mesothelioma appears to be correlated with genetics factors, radiation, viruses, and environmental carcinogens such as asbestos and erionite (2). The diagnosis is often based on cytological, histomorphological, immunohistochemical, and ultrastructural findings (1, 3, 4,
7). This paper describes a rare case of metastatic papillary mesothelioma in a Dachshund female dog.

\section{Case report}

A six-year-old female Dachshund was referred to the Small Animal Internal Medicine Service of Veterinary Hospital at Federal University of Uberlandia (UFU), Minas Gerais, Brazil. The animal presented intense abdominal distension caused by hemorrhagic effusions. Radiography and ultrasound examination did not reveal alterations. Cytological examination of the fluid revealed high cellularity with clusters of a population of large pleomorphic epithelioid cells featuring a prominent nucleolus and abundant basophilic cytoplasm. Those cells presented marked anisocytosis and anisokaryosis. Thus, the presumptive diagnosis was carcinoma.

As the dog continued presenting a large amount of abdominal hemorrhagic effusion, exploratory laparotomy 
was performed. During the procedure, only a focal alteration on the spleen's serosal surface color was observed and the organ was removed. On gross examination at the Animal Pathology Laboratory of UFU, a thin and white layer covering a limited area at the spleen's surface was observed. Histopathological examination revealed a thick splenic capsule and the presence of neoplastic cells with formed papillary structures arising from the capsule. Polygonal cells with a cytoplasm ranging from moderate to abundant, weakly basophilic, central nucleus, stippled chromatin, and prominent nucleolus could be discerned. The neoplastic cells presented moderate anisocytosis and anisokaryosis and moderate pleomorphism. Mitotic figures were not observed. The morphological features were consistent with undifferentiated neoplasm, indicating mesothelioma as the definitive diagnosis.

After surgery, the dog still presented abdominal effusion and developed thoracic and pericardial hemorrhagic effusion. Cytological examination was performed on both fluids and the same aforementioned characteristics were identified, in addition to a background of mixed inflammatory cells. Despite treatment with metronomic chemotherapy, the dog showed high production of thoracic and abdominal hemorrhagic effusion, which had to be drained every 3 days. The animal became highly dyspneic, stopped eating and sleeping, and the only comfortable position observed was the orthopneic position. Considering all these facts, the owners decided to euthanize the dog.

Necropsy was performed and revealed that both thoracic and abdominal cavities were filled with approximately $200 \mathrm{~mL}$ and $600 \mathrm{~mL}$ of sera-sanguineous fluid, respectively. There was no abnormal gross finding in the abdominal cavity. The pericardium was adhered to the ribs and covered by fibrotic plaques. A few firm white-togrey nodules, ranging in size from 0.1 to $0.3 \mathrm{~cm}$, were found in the lungs and diaphragm. Samples taken from the lungs and diaphragm were stained for routine histopathology at the Animal Pathology Laboratory of UFU, and immunohistochemistry (IHC) assay was performed by an external laboratory.

For the IHC assay, formalin-fixed paraffinembedded tissue sections ( $4 \mu \mathrm{m}$ thick) were prepared. Samples were deparaffinized in xylene and rehydrated in graded alcohols. Antigen retrieval was performed by heat using a pressure cooker (Pascal@, Dako) in citrate buffer (monohydrate citrate, $10 \mathrm{mM}, \mathrm{pH}$ 6). Endogenous peroxidase activity was blocked with hydrogen peroxide ready to use (Peroxide Block, Cell Marque, 925B-09). A blocking step was performed using Protein Block SerumFree (DAKO, X0909). Slides were incubated with the primary antibodies (vimentin, pancytokeratin, calretinin and thyroid transcription factor-1), overnight at $4{ }^{\circ} \mathrm{C}$ (Table 1). The reaction was revealed by Envision Dual Link (DAKO, K4065) and diaminobenzidine (DAKO, K3468). Slides were counterstained with Harris hematoxylin.
Cells displaying the same characteristics as the ones found in the spleen were observed in the lungs and diaphragm. Immunohistochemically, the tumor cells in these organs expressed vimentin, pancytokeratin, and calretinin and did not express thyroid transcription factor-1 (TTF-1). This led us to the diagnosis of metastatic epithelioid mesothelioma (5) (Table 1).

\section{Discussion}

Most animals with peritoneal mesothelioma receive veterinary care due to ascites. Other possible clinical symptoms include weight loss, anorexia, vomiting, and hemorrhage due to disseminated intravascular coagulation (6). In this case, the dog showed ascites and also developed a hemorrhagic thoracic effusion.

The first impression of cytological examination of abdominal fluids only provided evidence to support the diagnosis of malignant neoplasia: high cellularity with high pleomorphic cells, presented in clusters (Fig. 1). It is important to consider that cytological examination of fluids can be useful to differentiate between a neoplastic and an inflammatory/infection cause of ascites. However, it is extremely difficult to distinguish between reactive and neoplastic mesothelial cells based only on cytology alterations (6). It is well known that reactive mesothelial proliferations may mimic mesothelioma (or metastatic carcinoma) because reactive mesothelial proliferations may show high cellularity, numerous mitotic figures, cytologic atypia, necrosis, formation of papillary groups, and entrapment of mesothelial cells within fibrosis, mimicking invasion (5).

The primary histopathological examination revealed malignant proliferation of mesothelial cells in the spleen (Fig. 2). Histologic findings observed in this case were consistent with the observations reported in extant literature, that is, layers of dark plump cuboidal, columnar, or rounded, epithelioid cells with a distinct border and abundant pink cytoplasm supported by thin fibrovascular stroma (9). Furthermore, the epithelioid cells varied in size but were larger than reactive cells. The central nuclei were large, hyperchromatic, pleomorphic, had nucleolar organizer regions, and some cells were binucleate (4). Additionally, papillary mesotheliomas appeared as branching outgrowths of neoplastic cells supported by a central stromal core (6). All of these features were present in the lungs and diaphragm, thus supporting our final diagnosis of papillary mesothelioma.

Mesothelial cells express both cytokeratin and vimentin and, although rarely present in ovarian or renal carcinomas, this dual expression is more consistent with mesothelioma than with carcinoma $(6,8)$. Calretinin is demonstrated in nearly all epithelioid mesotheliomas. The staining is often strong and diffuse and is both nuclear and cytoplasmic; $5 \%-10 \%$ of lung adenocarcinomas are positive. Pancytokeratin is useful in the diagnosis of mesothelioma because virtually all epithelioid 
Table 1. Description of the antibodies.

\begin{tabular}{lccc}
\hline Antibody & Clone & Dilutions & Result \\
\hline Vimentin (Invitrogen $®)$ & V9 & $1: 200$ & Positive \\
CK PAN* (Invitrogen $®)$ & AE1AE3 & $1: 200$ & Positive \\
Calretinin (Spring $®)$ & Polyclonal & $1: 100$ & Positive \\
TTF-1** (Spring () & Polyclonal & $1: 100$ & Negative \\
\hline
\end{tabular}
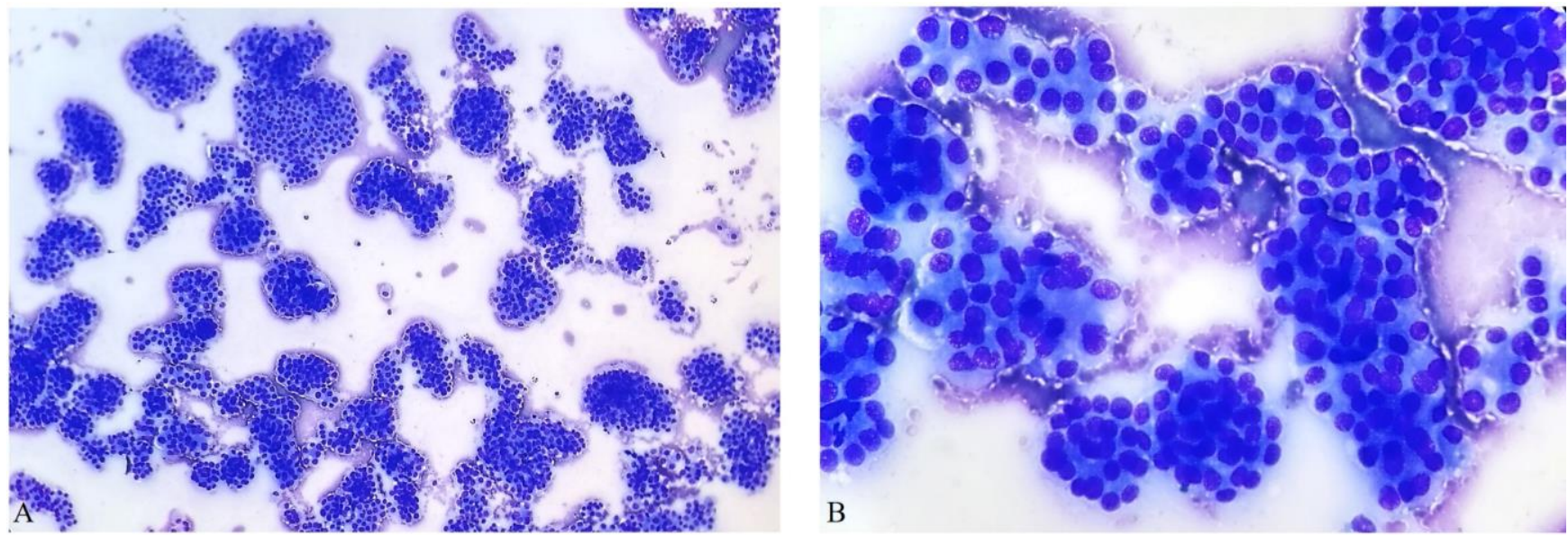

Figure 1. Cytological examination of the abdominal fluid. Neoplastic cells presented in clusters, high cellularity (A. 100X Panótico Rápido - Laborclin $\left.{ }^{\circledR}\right)$. Population of large pleomorphic epithelioid cells with prominent nucleolus and abundant basophilic cytoplasm, stippled chromatin, marked anisocytosis and anisokaryosis (B. 400X Panótico Rápido - Laborclin®).

mesotheliomas will produce positive results. About $75 \%-$ $85 \%$ of lung adenocarcinomas show nuclear positivity to TTF-1 and it is not expressed in mesotheliomas; therefore, it is used to differentiate between these two neoplasms (8).

In our case study, the cells were positive for vimentin, pancytokeratin, and calretinin expression and negative for TTF-1 expression. We also confirmed metastatic papillary mesothelioma in the diaphragm (Fig. 3) and lungs (Fig. 4). Metastasis to the diaphragm, which is an unusual site, suggests the need for investigation of other primary sites.

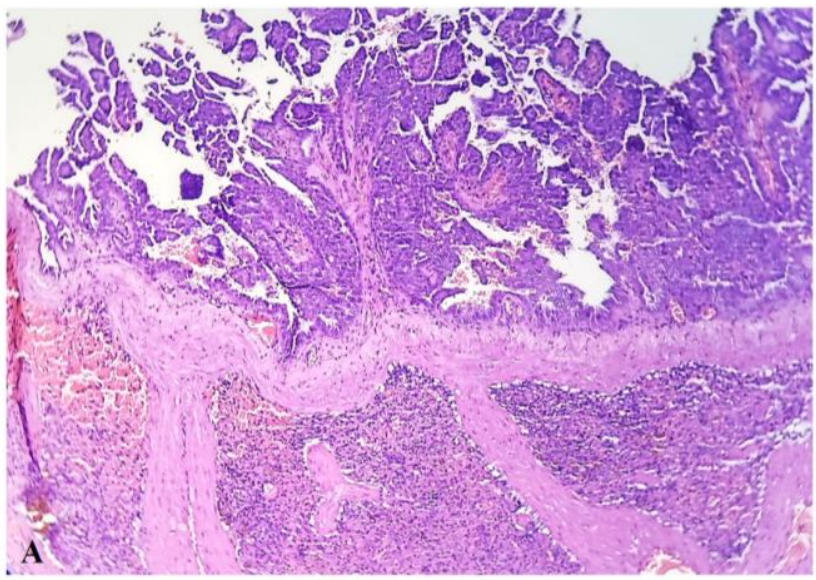

In conclusion, we describe a rare case of metastatic epithelioid mesothelioma in a female Dachshund dog, with both abdominal and thoracic cavities affected. In this case, we used cytology examination as the first exam, and that led us to primary differential diagnosis between carcinoma and mesothelioma. The immunohistochemistry examination was considered essential to determine that, in fact, the dog presented a rare type of papillary mesothelioma, which had metastasized to the diaphragm and lungs.

Figure 2. Papillary mesothelioma. Neoplastic cells with formed papillary structures arising from the splenic capsule (A. HE 100X). Epithelioid cells with an abundant pink cytoplasm supported by a thin fibrovascular stroma. The cells vary in size but are larger than reactive cells. The central nuclei are large, hyperchromatic, pleomorphic, and present stippled chromatin, and prominent nucleolus. Moderate anisocytosis and anisokaryosis, and moderate pleomorphism. Mitotic figures were not observed

(B.

$\mathrm{HE}$ 400X). 


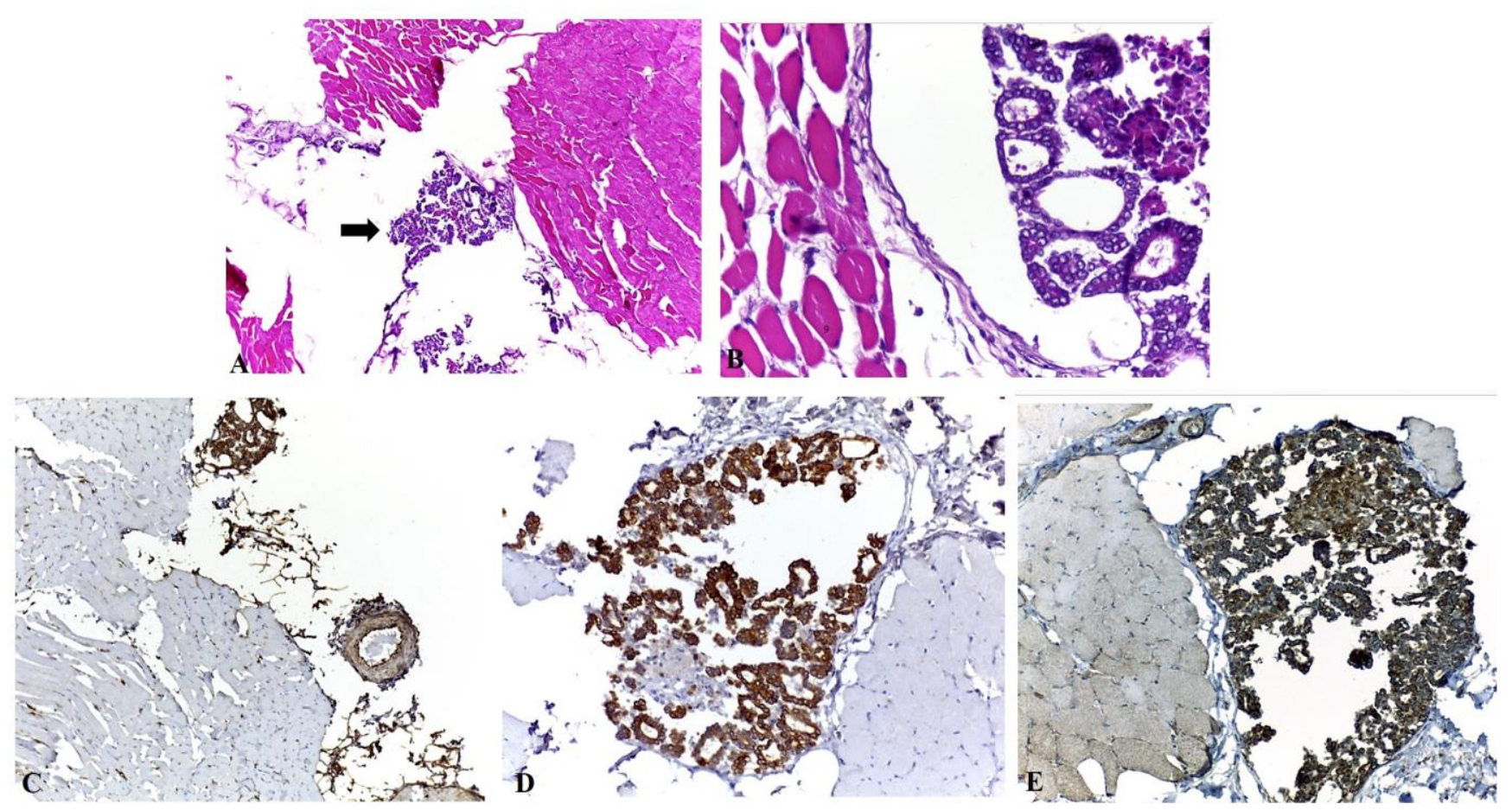

Figure 3. Metastatic papillary mesothelioma in the diaphragm. Neoplastic cells in the diaphragm (arrow) (A. HE 40X). Epithelioid cells with an abundant pink cytoplasm. The cells vary in size but are large. Moderate anisocytosis and anisokaryosis, and moderate pleomorphism (B. HE 200X). Neoplastic cells were positive for vimentin (C. IHC 40X), pancytokeratin (D. IHC 200X), and calretinin (E. IHC 200X).

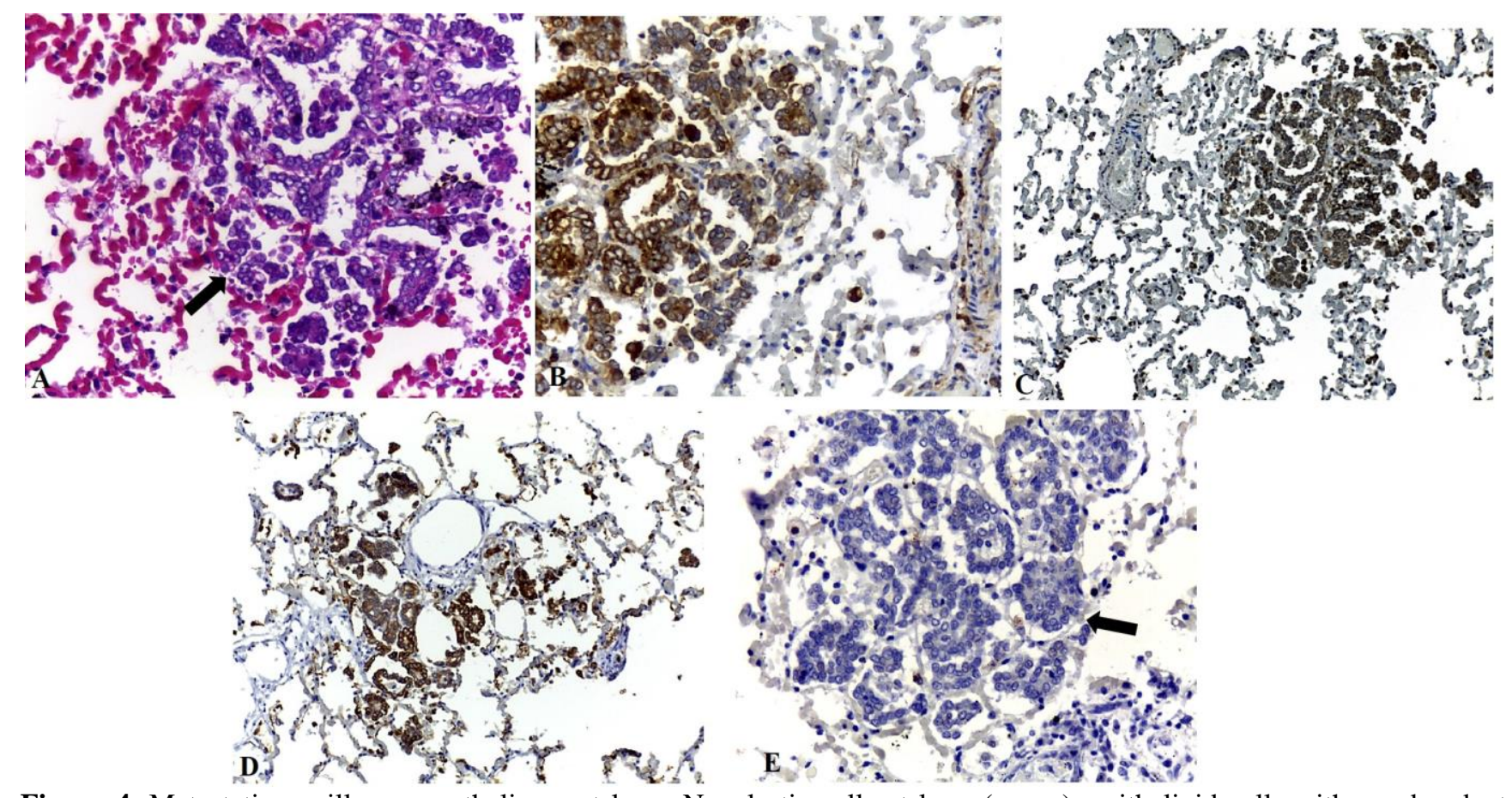

Figure 4. Metastatic papillary mesothelioma at lung. Neoplastic cells at lung (arrow), epithelioid cells with an abundant pink cytoplasm. The cells vary in size but are large. Moderate anisocytosis and anisokaryosis, moderate pleomorphism (A. HE 400X). Neoplastic cells were positive for vimentin (B. IHC 400X), calretinin (C. IHC 100X), and pancytokeratin (D. IHC 200X). Neoplastic cells were negative for TTF-1 (arrow) (E. IHC 200X). 


\section{References}

1. Avakian A, Alroy J, Rozanski E, Keating J, Rosenberg A. Lipid-rich pleural mesothelioma in a dog. J Vet Diagn Invest. 2008;20(5):665-7.

2. Carbone M, Kratzke RA, Testa JR. Seminars in oncology. 2002;29(1):2-17.

3. Harbison ML, Godleski JJ. Malignant mesothelioma in urban dogs. Vet Pathol. 1983;20(5):531-40.

4. Head KW, Else RW, Dubielzig RR. Tumors of serosal surfaces. In: DJ Meuten, editor. Tumors in domestic animals. Ames, Iowa; Iowa State Press, Ames; 2002. p. 477-78.

5. Husain AN, Colby TV, Ordonez NG, Allen TC, Attanoos RL, Beasley M B, Butnor KJ, Chirieac LR, Churg AM, Dacic S, Galateau-salle F, Gibbs A, Gown AM, Krausz T, Litzky LA, Marchevsky A, Nicholson AG, Roggli VL, Sharma AK, Travis WD, Walts AE, Wick MR. Guidelines for pathologic diagnosis of malignant mesothelioma 2017 update of the consensus statement from the International Mesothelioma Interest Group. Arch Pathol Lab Med. 2017;142(1):89-108.

6. Munday JS, Löhr CV, Kiupel M. Tumors of the Alimentary Tract. In: DJ Meuten, editor. Tumors in domestic animals. Ames, Iowa: John Wiley \& Sons Inc.; 2017. p. 499-601.

7. Ordóñez NG. The diagnostic utility of immunohistochemistry and electron microscopy in distinguishing between peritoneal mesotheliomas and serous carcinomas: a comparative study. Modern pathology. 2006;19(1):34-48.

8. Painter, JT, Clayton, NP, Herbert, RA. Useful immunohistochemical markers of tumor differentiation. Toxicol Pathol. 2010;38(1):131-41.

9. Uzal FA, Plattner BL, Hostetter JM. Alimentary System. In: Maxie G, editor. Jubb, Kennedy \& Palmer's Pathology of Domestic Animals. St. Louis: Elsevier; 2015. p. 2-257. 